\title{
A neural predictor of cultural popularity
}

\author{
Gregory S. Berns *, Sara E. Moore \\ Economics Department and Center for Neuropolicy, Emory University, Atlanta, GA 30322, USA
}

Received 7 February 2011; accepted 5 May 2011

Available online 8 June 2011

\begin{abstract}
We use neuroimaging to predict cultural popularity — something that is popular in the broadest sense and appeals to a large number of individuals. Neuroeconomic research suggests that activity in reward-related regions of the brain, notably the orbitofrontal cortex and ventral striatum, is predictive of future purchasing decisions, but it is unknown whether the neural signals of a small group of individuals are predictive of the purchasing decisions of the population at large. For neuroimaging to be useful as a measure of widespread popularity, these neural responses would have to generalize to a much larger population that is not the direct subject of the brain imaging itself. Here, we test the possibility of using functional magnetic resonance imaging (fMRI) to predict the relative popularity of a common good: music. We used fMRI to measure the brain responses of a relatively small group of adolescents while listening to songs of largely unknown artists. As a measure of popularity, the sales of these songs were totaled for the three years following scanning, and brain responses were then correlated with these "future" earnings. Although subjective likability of the songs was not predictive of sales, activity within the ventral striatum was significantly correlated with the number of units sold. These results suggest that the neural responses to goods are not only predictive of purchase decisions for those individuals actually scanned, but such responses generalize to the population at large and may be used to predict cultural popularity.
\end{abstract}

(C) 2011 Published by Elsevier Inc. on behalf of Society for Consumer Psychology.

Keywords: fMRI; Neuroeconomics; Neuromarketing; Music; Prediction

\section{Introduction}

How can we predict popularity? Although superficially a trivial question, the desire for popularity consumes a great portion of the lives of many youths and adults. More than the superficial teenager's quest for popularity, being popular is a marker for social status. Consequently, popularity would seem to confer a reproductive advantage in the evolution of the human species, thus explaining its importance to humans. Such importance extends to economic success as well because goods and services that are popular command higher prices. Although there are good economic and evolutionary rationales for pursuing popularity, predicting who or what becomes popular is a challenging problem. Even so, the ability to predict

\footnotetext{
* Corresponding author at: 1602 Fishburne Dr., Emory University, Atlanta, GA 30322, USA.

E-mail address: gberns@emory.edu (G.S. Berns).
}

popularity is a valuable skill that also can translate into economic success.

In the domain of economic goods, traditional approaches to forecasting popularity rely on standard marketing techniques. These include focus groups, questionnaires, simulated choice tests, and market tests. More recently, however, the widespread use of neuroimaging has raised the possibility of using functional magnetic resonance imaging (fMRI) in the marketing process (Ariely \& Berns, 2010). Neuroeconomic research suggests that activity in reward-related regions of the brain, notably the orbitofrontal cortex and ventral striatum is predictive of future purchasing decisions of the individuals who are scanned (Hare, O’Doherty, Camerer, Schultz, \& Rangel, 2008; Knutson, Rick, Wimmer, Prelec, \& Loewenstein, 2007; Plassmann, O’Doherty, \& Rangel, 2007; Plassmann, O’Doherty, Shiv, \& Rangel, 2008). For neuroimaging to be useful in either a marketing or branding application, however, these neural signals would need to generalize to a larger group of individuals who themselves were not the direct object of 
brain scanning. Currently, it is unknown whether the neural signals of a small group of individuals are predictive of the purchasing decisions of the population at large.

Neuroimaging is often touted as a hot new tool for branding (Lindstrom, 2008). Although branding and advertising have been considered in a few neuroimaging papers (Kenning \& Plassmann, 2008; Lee, Broderick, \& Chamberlain, 2007; Yoon, Gutchess, Feinberg, \& Polk, 2006), it is still unknown whether neuroimaging can prospectively reveal whether a particular ad or brand campaign will be effective. In a wellknown Coke-Pepsi study, participants who described themselves as Coke-drinkers showed significant activation in the hippocampus and right DLPFC when they were cued about the upcoming drink of Coke (McClure et al., 2004). Self-described Pepsi-drinkers did not have this response. In the absence of brand information, there was no significant difference in preference during a test-taste. This study suggested that any differences in the neural response to the two brands must be culturally derived. Although these results demonstrate that branding does affect brain responses to nominally similar goods, the question of whether brand effectiveness can be predicted in advance remains an open question.

To demonstrate the efficacy of an fMRI study for branding, three conditions must be met. First, the study participants-i.e. the cohort of individuals who are actually scanned - should be representative of the population that is the target of a brand campaign. Second, to truly test whether the neural signals are predictive of brand effectiveness, the scanning must be done before the campaign is launched. Third, metrics of brand effectiveness must be readily available for the target population. For example, these might include sales data, web page views, downloads, internet searches, etc. Finally, although not strictly a condition, it is an open question as to what should actually be scanned during fMRI. If the product can be consumed in the scanner, then the product itself becomes the target. Alternatively, an ad or branding campaign might be presented in the scanner, in which case an abstract association between an ad and a product becomes the scanned target.

One product that meets these requirements is music. Everyone has musical preferences, and most people spend money on this product. Thus, it is straightforward to find people to scan who are representative of the music-consuming public, which is almost everyone. Second, the rise of sites like myspace.com has created a large repository of music which is largely unadvertised and unbranded. Because much of this music is provided directly by the artist, it can be used well in advance of any ad campaign; moreover, the band is the brand. Third, metrics of music success are simple and straightforward: downloads, sales, and ticket receipts. Finally, music is ideally suited to scanning because the act of listening to it is the same as consuming it. Thus, imaging the neural response to music is a direct measure of the consumption experience. Subsequent success is then a combination of quality, branding, and marketing.

In a previous study of adolescents, we measured the interaction of social influence in the form of popularity ratings with the consumption experience of music (Berns, Capra,
Moore, \& Noussair, 2010). Using fMRI, we found that although an individual's musical preferences were strongly correlated with activity in the caudate nucleus, the effect of social information varied between participants. The tendency to change one's evaluation of a song was positively correlated with activation in the anterior insula and anterior cingulate, two regions that are associated with physiological arousal and negative affective states. While this earlier study examined the effect of popularity information on individual preferences, here we report a longitudinal analysis in which we examine the relationship between brain responses and popularity of music from the other direction: do neural responses to music in an fMRI study predict subsequent commercial success of the song and artist?

\section{Material and methods}

A total of 32 adolescent participants were studied. Five were excluded from the fMRI analyses due to either excessive movement or susceptibility artifacts. Although this was a relatively high exclusion rate compared to adult studies, it was comparable to previous fMRI studies in children and adolescents, who tend to move more than adults (Galvan et al., 2006). Prior to the experiment, they were screened for the presence of medical and psychiatric diagnoses, and none were taking medications. There were 14 female and 13 male participants between the ages of 12 and 17.9 (mean 14.6). Fifteen were Caucasian, eight were African-American, one was Hispanic, and three were "Other." The primary stimuli used were 15-s clips from songs downloaded from MySpace.com. Songs were downloaded between October 23 and November 8, 2006. In order to minimize the possibility that participants would recognize the artists, songs from unsigned musicians or relatively unknown artists were used. A total of 20 songs were downloaded in each of the following genres: Rock, Country, Alternative/ Emo/Indie, Hip-Hop/Rap, Jazz/Blues, and Metal (identified by the MySpace category). At the time of download, the number of times each song had been played was recorded, and this was used to calculate the popularity of each song among MySpace users. Each song was converted from MP3 to WAV format and a 15-s clip was extracted that included either the hook or chorus of the song. These 15 -s clips were subsequently used in the experiment.

At the beginning of each session, individuals' rankings of musical genres were elicited. Participants were provided with a list of the six musical genres, and were instructed to rank the genres from 1 ("the type you like the best") to 6 ("the type you like the least"). Each participant's top three genres were subsequently used in the experiment. Emory University's Institutional Review Board approved all procedures. Individuals then entered the scanner, and the total scan time was approximately one hour. The scanning was performed on a Siemens 3T Trio. Each subject received a T1-weighted structural image $(\mathrm{TR}=2600 \mathrm{~ms}, \mathrm{TE}=3.93 \mathrm{~ms}$, flip angle $=8,224 \times 256$ matrix, 176 sagittal slices, $1 \mathrm{~mm}$ cubic voxel size), a DTI scan $(\mathrm{TR}=6500 \mathrm{~ms}, \mathrm{TE}=90 \mathrm{~ms}$, flip angle $=90, \mathrm{FOV}=220 \mathrm{~mm}$, $128 \times 128$ matrix, 34 axial slices, $1.7 \times 1.7 \times 2.5 \mathrm{~mm}$ voxel size, 
6 sets of 12 directional $b=1000$ and $1 b=0$ images), and 3 functional runs of BOLD-weighting $(\mathrm{TR}=2000 \mathrm{~ms}, \mathrm{TE}=31 \mathrm{~ms}$, flip angle $=90, F O V=192 \mathrm{~mm}, 64 \times 64$ matrix, 28 axial slices, $3 \mathrm{~mm}$ cubic voxel size). Each individual participated in 60 trials. Each trial was divided into two stages; in each stage the subject listened to the same 15-s song clip (Fig. 1a). During stage 1, no popularity information was shown. After listening, subjects were required to rate the song based on (a) how familiar it was and (b) how much they liked it. Both ratings used a 1-5 star scaling system. To prevent the subject from passively accepting a default rating, each rating screen began with 0 stars, which could not be accepted as a final selection. After the rating was entered, stage 2 of the trial took place. The clip was played again, after which the subject provided another likability rating. Twenty songs in each of the subject's top-three genres were presented in random order throughout the experiment. In $2 / 3$ of the trials, during the second listen, the song's popularity was displayed in the 1-5 star scaling system. The 40 trials in which the popularity display appeared were sequenced randomly among the 60 trials. Only brain activation data from the first listening period was used in the subsequent analysis. As an incentive to accurately reveal their song preferences, each subject received a CD with their top-rated songs.

Nielsen SoundScan was used as the source of post factum popularity information over the three years since the songs were originally chosen for the study. The SoundScan database was searched for information on the performers of each of the 120 songs in the study. Sales data were available for 87 songs,

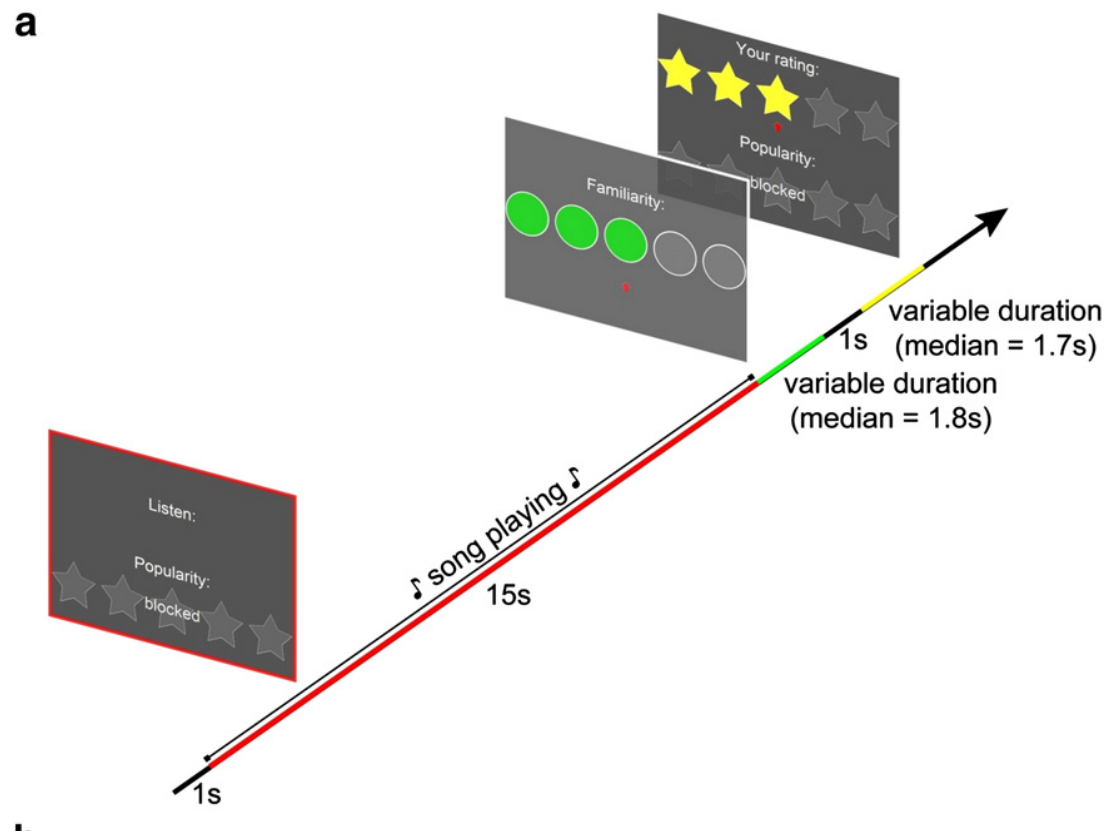

b

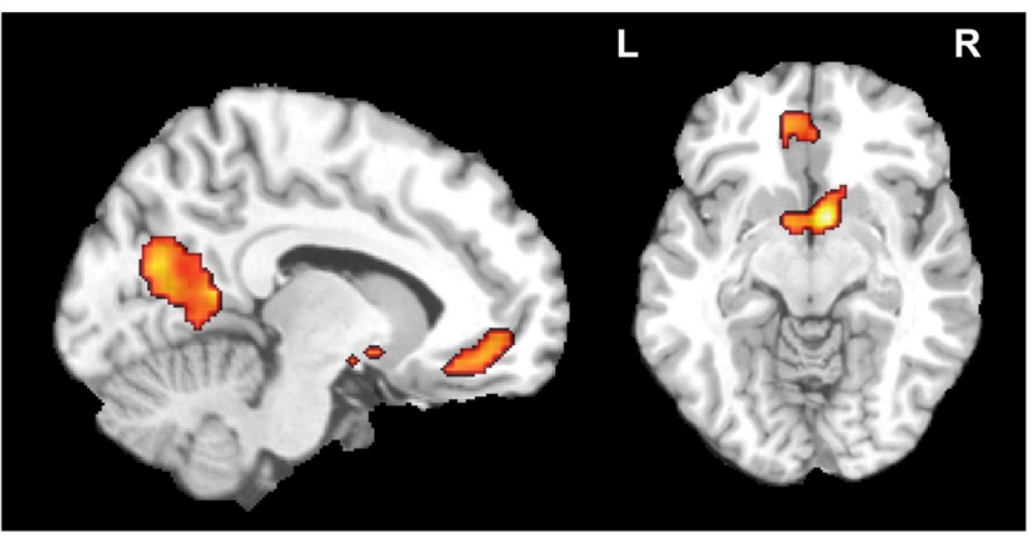

Fig. 1. Design of popular music experiment and brain regions correlated with the average likability of each song. a) Timing of events for a typical trial. Each song was played for $15 \mathrm{~s}$. Each participant heard 60 songs from their favorite 3 genres. Following the song, participants rated the song for familiarity and likability. The trial was then repeated with the average popularity shown on $2 / 3$ of the trials and blocked for $1 / 3$ of the trials. Only the initial listening period (red) was used for subsequent analyses. The first listening period for each of the songs was modeled separately, for a total of sixty 15 second variable duration events for each participant. Secondlevel models for each of the 120 songs were constructed as one-sample t-tests from the contrast images of the first listening period from the first-level model. A thirdlevel model was then built using the positive contrast images from the second-level model. This model also included a covariate of the first likability rating for each song, averaged over the participants who heard that song. b) Brain regions positively correlated with the average likability of the song from the third-level model $(\mathrm{p}<0.005$, cluster extent $\geq 56$, yielding whole-brain FDR $<0.05)$ were limited to three areas: cuneus, orbitofrontal cortex, and ventral striatum. 
and this data was extracted from the SoundScan database during May 2010. The obtained metric aggregated all recorded sales of the song from release through May 2010, including singles, albums, and compilations.

Preprocessing of the fMRI data was executed in SPM5 (Functional Imaging Laboratory, UCL, London). The preprocessing pipeline consisted of slice timing correction, motion correction, spatial normalization, and smoothing (with an $8 \mathrm{~mm}$ Gaussian kernel). A first-level GLM was constructed in SPM5 for each of the 27 participants. The first listening period for each of the 60 songs was modeled separately, for a total of sixty 15-second variable duration events. All of the second listening periods, also $15 \mathrm{~s}$ variable duration events, were collapsed into a single condition for each run. All three variable duration rating phases of the trial (familiarity, first likability, and second likability) were also collapsed into one condition to model the act of rating including the button presses. The motion parameters were also included in the model as an effect of non-interest. Second-level models for each of the 120 songs were constructed as one-sample t-tests in SPM5 using contrast images of the first listening period from the first-level model above. Since every participant did not hear every song, the number of contrast images in each of these second-level models ranged from 3 to 23 . A third-level model, also a one-sample t-test, was then built in SPM5 using the positive contrast images from the second-level model above. This model also included a covariate of the first likability rating for each song, averaged over the participants who heard that song. We used the likability covariate to identify ROIs. Statistical thresholds were determined based on the estimated smoothness of the 3rd-level contrasts. Using the AlphaSim routine in AFNI, we estimated the combination of height and extent thresholds that yielded a whole-brain FDR $<0.05$ (10,000 iterations). First, white matter and CSF were masked out using the SPM probabilistic gray matter map. With a gray matter probability $>0.6$, this results in a mask that retains most gray matter while effectively eliminating most white matter and CSF, which would otherwise inflate the required cluster size. Second, we used 3dFWHMx to estimate the image smoothness from the square root of the masked SPMgenerated ResMS image and input into AlphaSim. Finally, using a voxel level threshold of $\mathrm{p}<0.005$, the extent threshold that yielded a cluster level alpha of 0.05 was determined to be $\mathrm{k} \geq 56$ (Logan \& Rowe, 2004; Zhang, Nichols, \& Johnson, 2009). The $60 \%$ gray matter mask was applied to all contrasts before using these thresholds. Only three brain regions showed a significant correlation between activation and average song likability: cuneus, orbitofrontal cortex (OFC) and ventral striatum/nucleus accumbens (NACC) (Fig. 1b). The activations for each song were then extracted from these regions of interest (ROIs) for subsequent analyses with song sales data. This approach ensures that the ROIs are defined independently from the variable of interest (song sales).

\section{Results}

The vast majority of songs in our sample were not commercially successful. The distribution of sales exhibited a long tail (Fig. 2a), with only three songs' albums meeting the industry standard for "gold" (500,000 units). Given the large number of songs released annually, this is not surprising. To normalize the distribution, sales data were log-transformed for subsequent analyses. First, we checked if either of the subjective song ratings were predictive of future sales, but neither of the two average ratings obtained for each song was correlated with sales data (likability: $\mathrm{R}=0.110, \mathrm{p}=0.313$; familiarity: $\mathrm{R}=0.106$, $p=0.330$ ), nor was the average genre ranking of each song $(p=0.102)$. This indicates that simple subjective reports collected from study participants may not be good predictors of commercial success.

Although subjective ratings of songs did not correlate with future sales, the activation within the NACC did (Fig. 2b). $\log ($ sales +1$)$ was significantly correlated with the average activation in NACC during the 15 -sec listening period $(\mathrm{R}=0.32$, $\mathrm{p}=0.004$ ). To see which part of the 15 -sec period was responsible for this correlation (e.g. initial or final reactions), we tested an alternative model with the listening period divided into three 5-sec segments. None of these three segments exhibited a greater correlation to sales than the whole $15-\mathrm{sec}$ period. This indicates that the mechanism driving the correlation between NACC activity and sales was integrated over the entire listening period.

To further understand the interrelationship between song likability, NACC activity, and sales, we constructed a structural equation model (SEM) (Fig. 3a). The SEM was based on known anatomical connections between the OFC and NACC and their relationships to subjective likability and purchase decisions (Chib, Rangel, Shimojo, \& O'Doherty, 2009; Knutson, et al., 2007; Montague \& Berns, 2002; O’Doherty, Kringelbach, Rolls, Hornak, \& Andrews, 2001; Plassmann et al., 2007; Rolls, 2000). Consistent with this literature, the average song likability had significant path coefficients to both the OFC and NACC, and because of the direct connection between OFC and NACC, this path was also significant. However, the final pathway linking the brain to album sales was mediated only through the NACC. When these relationships were visualized, it became clear that "hit" songs did not result from a specific combination of NACC and OFC activity, but that "non-hits" were associated with a combination of both low OFC and low NACC activity (Fig. 3b). This relationship was quantified through logistic regressions on different hit/non-hit thresholds of sales (Fig. 3c). With thresholds in the range of 15,000 to 35,000 units sold, the logistic model achieved reasonable accuracy in correctly classifying hits and non-hits. For example, with a hit-threshold of 15,000 units, the logistic model correctly classified $80 \%$ of the non-hits; however, this came at a cost of missing true hits (but still correctly classified $30 \%$ of the hits). To test the possibility that consistency of brain responses might also be predictive of sales, we formulated a model that included a term for the reciprocal of the variance of the brain response across subjects that heard each song; however, this term was not significant $[\mathrm{F}(1,83)=0.28, \mathrm{p}=0.597]$.

\section{Discussion}

Our results demonstrate that not only are signals in rewardrelated regions of the human brain predictive of individual 
a
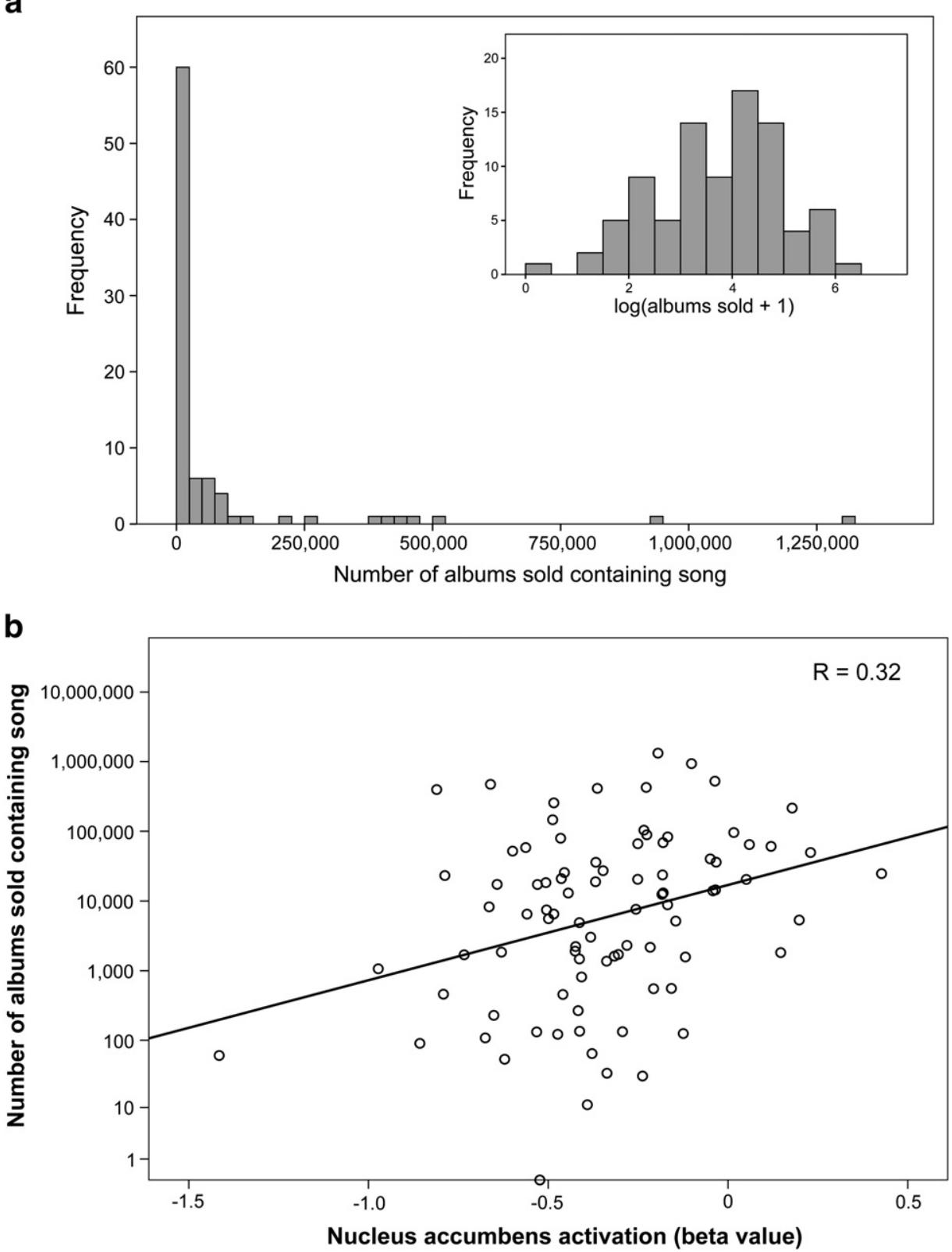

Fig. 2. Distribution of number of albums sold and correlation with nucleus accumbens activation. a) Song sales were determined by sales data reported by Nielsen SoundScan from the album release through May 2010. The distribution was positively skewed, indicating that most songs did not sell many units and with a long tail to the right. Log-transformation of the sales data normalized this distribution for subsequent correlations (inset). b) The log of the number of units sold of a song was significantly correlated with the average nucleus accumbens [MNI coordinates: $9,6,-9 ; \mathrm{p}<0.005,59$ voxels] activation during the listening of the song $(\mathrm{R}=0.32$, $\mathrm{p}=0.004)$. Exclusion of the far left outlier resulted in a decreased correlation but which was still significant $(\mathrm{R}=0.27, \mathrm{p}=0.013)$.

purchase decisions, they are also modestly predictive of population effects. While the nascent field of neuromarketing has made claims to this effect, truly prospective data has been lacking (Ariely \& Berns, 2010). Surprisingly, our data suggest some validity to these claims. Why might this be? If the specific cohort of participants in an imaging study is representative of a particular population, then it follows that the results should generalize. When it comes to music, however, it may be difficult to know on which dimensions of a population to match (e.g. age, gender, SES, region). The Recording Industry Association of America (RIAA) estimates that the age range of our cohort accounts for approximately $20 \%$ of music sales (www.riaa.com/ keystatistics.php). To test whether the musical tastes of our cohort were representative of the population, we compared our cohort's pre-scan genre rankings to the 2009 Nielsen sales by category and found a significant correlation (Kendall's $\tau=-0.733, \mathrm{p}=0.05$; assuming that our hip-hop category is equivalent to Nielsen's R\&B category), showing that our cohort had similar tastes as the national population.

Our results also raise the question of why the brain activation was predictive of future sales but the self-reported likability ratings were not. One possibility is that the questions were not 

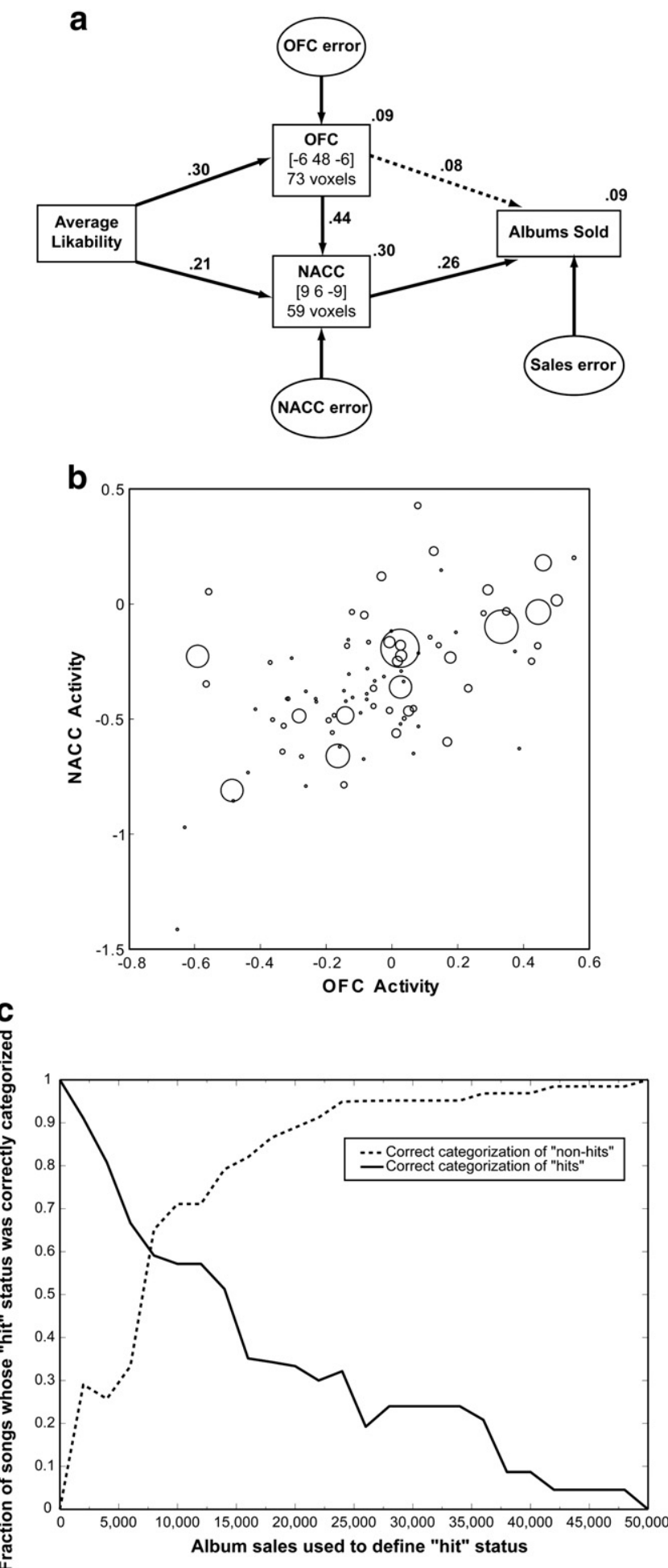

Fig. 3. Structural equation model linking likability ratings to albums sold through OFC and NACC, and logistic regression to categorize "hits." a) Although the average likability of each song was not directly correlated with the number of albums sold, the relationship was moderated by activity in the OFC and NACC. All path coefficients and main effects were significant $(\mathrm{p}<0.05)$ except that linking OFC to albums sold. b) The sales of each are represented by the area of each circle and plotted as a function of both OFC and NACC activity. Although the "hits" (big circles) are scattered throughout the activation space, there are a higher proportion of "non-hits" (small circles) toward the lower left. c) To test the ability to correctly categorize "hits" and "non-hits," logistic regressions were performed. The threshold of hit vs. non-hit was varied from 1000 to 50,000 units. Thresholds in the range 15,000 to 35,000 correctly categorize a sizable fraction of hits while also correctly rejecting most non-hits. adequately specified to differentiate future success (Cronbach \& Meehl, 1955). A more detailed debriefing of the reactions to each song might have yielded better predictive data. For example, a choice-based conjoint model might have been superior to simple rankings (Green \& Srinivasan, 1990; Griffin \& Hauser, 1993). Another possibility is that above a certain quality threshold, songs are too similar to prospectively differentiate them, but slight differences in quality become magnified in superstar markets (Rosen, 1981). Although our results do not invalidate any of these approaches, they do suggest that brain imaging can augment them. The SEM showed that even though likability was not directly correlated with future sales, the OFC and NACC moderated this relationship as essentially hidden variables within the brain. Asking an individual how much they like something requires several cognitive operations, including the initial processing of the stimulus, referencing similar items with which the individual has experience, projection of future utility, all of which may be subject to framing effects of the experiment. In contrast, brain responses in reward-related regions are likely to reflect sub-conscious processes and may yield measurements that are less subject to cognitive strategies. This would be especially true during the consumption of music, which occurred during the listening phase of our experiment. Thus, while the act of rating something requires metacognition, the brain response during the consumption of the good does not, and the latter may prove superior to rating approaches.

Although our data raise the possibility of predicting future popularity in the form of commercial sales, the actual performance of such a model depended on the definition of a "hit." The scarcity of true hits (e.g. 500,000 units) in our sample, underscores the difficulty in evaluating a hit-predictor and confirms the previously noted shift toward superstars (Krueger, 2005). The logistic model performed well in identifying nonhits, which may itself be valuable information, but given the widely varying marketing approaches that are invested in bands (Vogel, 2007), it is surprising that we found any predictive power at all. The fact that we used a wide variety of songs in different genres certainly made the prediction of hits more difficult. A more focused presentation of songs, perhaps within a single genre and pre-screened for minimal quality, would increase the likelihood of hit-prediction. A more targeted group of study participants that is representative of a particular music consuming demographic might also increase predictive power. However, predicting hit-songs may always be a particularly difficult task. A recent study of internet searches found good predictability for revenue of movies and video games but less so for music (Goel, Hofman, Lahaie, Pennock, \& Watts, 2010), which makes our results even more surprising and refutes the idea that hits are random (Bielby \& Bielby, 1994).

Our results may also have implications for branding. In commercial music, the band is the brand. We calculated commercial success based on the number of units sold, but this number included all sources of a particular song. As a result, the sales numbers were dominated by album sales, which of course contain many songs and may been heavily influenced by the band/artist reputation (i.e. the band brand). It is hard to know what marketing efforts might have been done to promote a 
band. However, it has been estimated that only $10 \%$ of new releases end up making a profit for a record label (Vogel, 2007). Consequently, marketing and branding efforts tend to be minimal until a band shows signs of popularity. With more and more artists having access to quality production equipment and being able to release songs directly to the public, neuroimaging tools may have real utility to help labels decide how to invest limited marketing and branding resources.

\section{Acknowledgments}

This work was supported by grants from the National Institute for Drug Abuse, the National Science Foundation, the Air Force Office of Scientific Research, and the Office of Naval Research.

\section{References}

Ariely, D., \& Berns, G. S. (2010). Neuromarketing: The hope and hype of neuroimaging in business. Nature Reviews Neuroscience, 11, 284-292.

Berns, G. S., Capra, C. M., Moore, S., \& Noussair, C. (2010). Neural mechanisms of the influence of popularity on adolescent ratings of music. NeuroImage, 49, 2687-2696.

Bielby, W. T., \& Bielby, D. D. (1994). “All hits are flukes”: Institutionalized decision making and the rhetoric of network prime-time program development. American Journal of Sociology, 99(5), 1287-1313.

Chib, V. S., Rangel, A., Shimojo, S., \& O’Doherty, J. P. (2009). Evidence for a common representation of decision values for dissimilar goods in human ventromedial prefrontal cortex. The Journal of Neuroscience, 29(39), $12315-12320$.

Cronbach, L. J., \& Meehl, P. E. (1955). Construct validity in psychological tests. Psychological Bulletin, 52, 281-302.

Galvan, A., Hare, T. A., Parra, C. E., Penn, J., Voss, H., Glover, G., et al. (2006). Earlier development of the accumbens relative to orbitofrontal cortex might underlie risk-taking behavior in adolescents. The Journal of Neuroscience, 26, 6885-6892.

Goel, S., Hofman, J. M., Lahaie, S., Pennock, D. M., \& Watts, D. J. (2010). Predicting consumer behavior with Web search. Proceedings of the National Academy of Sciences of the United States of America, 107(41), 17486-17490.

Green, P. E., \& Srinivasan, V. (1990). Conjoint analysis in marketing: New developments with implications for research and practice. Journal of Marketing, 54(4), 3-19.
Griffin, A., \& Hauser, J. R. (1993). The voice of the customer. Marketing Science, 12(1), 1-27.

Hare, T. A., O’Doherty, J., Camerer, C. F., Schultz, W., \& Rangel, A. (2008) Dissociating the role of the orbitofrontal cortex and the striatum in the computation of goal values and prediction errors. The Journal of Neuroscience, 28(22), 5623-5630.

Kenning, P. H., \& Plassmann, H. (2008). How neuroscience can inform consumer research. IEEE Transactions on Neural Systems and Rehabilitation Engineering, 16(6), 532-538.

Knutson, B., Rick, S., Wimmer, G. E., Prelec, D., \& Loewenstein, G. (2007). Neural predictors of purchases. Neuron, 53, 147-156.

Krueger, A. B. (2005). The economics of real superstars: The market for rock concerts in the material world. Journal of Labor Economics, 23(1), $1-30$.

Lee, N., Broderick, A. J., \& Chamberlain, L. (2007). What is neuromarketing? A discussion and agenda for future research. International Journal of Psychophysiology, 63, 199-204.

Lindstrom, M. (2008). Buyology. Truth and lies about why we buy. New York: Doubleday.

Logan, B. R., \& Rowe, D. B. (2004). An evaluation of thresholding techniques in fMRI analysis. Neurolmage, 22, 95-108.

McClure, S. M., Li, J., Tomlin, D., Cypert, K. S., Montague, L. M., \& Montague, P. R. (2004). Neural correlates of behavioral preference for culturally familiar drinks. Neuron, 44, 379-387.

Montague, P. R., \& Berns, G. S. (2002). Neural economics and the biological substrates of valuation. Neuron, 36, 265-284.

O’Doherty, J., Kringelbach, M. L., Rolls, E. T., Hornak, J., \& Andrews, C. (2001). Abstract reward and punishment representations in the human orbitofrontal cortex. Nature Neuroscience, 4(1), 95-102.

Plassmann, H., O’Doherty, J., \& Rangel, A. (2007). Orbitofrontal cortex encodes willingness to pay in everyday economic transactions. The Journal of Neuroscience, 27(37), 9984-9988.

Plassmann, H., O’Doherty, J., Shiv, B., \& Rangel, A. (2008). Marketing actions can modulate neural representations of experienced pleasantness. Proceedings of the National Academy of Sciences of the United States of America, 105(3), $1050-1054$

Rolls, E. T. (2000). The orbitofrontal cortex and reward. Cerebral Cortex, 10, 284-294.

Rosen, S. (1981). The economics of superstars. American Economic Review, 71(5), 845-858.

Vogel, H. L. (2007). Entertainment industry economics. A guide for financial analysis (7th ed.). Cambridge: Cambridge University Press.

Yoon, C., Gutchess, A. H., Feinberg, F., \& Polk, T. A. (2006). A functional magnetic resonance imaging study of neural dissociations between brand and person judgments. Journal of Consumer Research, 33, 31-40.

Zhang, H., Nichols, T. E., \& Johnson, T. D. (2009). Cluster mass inference via random field theory. NeuroImage, 44, 51-61. 